\title{
Genome-Wide Dissection of the CRF Gene Family in Brassica napus Indicates that BnaCRF8s Specifically Regulate Root Architecture and Phosphate Homeostasis against Phosphate Fluctuation in Plants
}

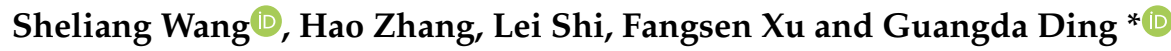 \\ Microelement Research Centre, National Key Laboratory of Crop Genetic Improvement, Key Laboratory of \\ Arable Land Conservation (Middle and Lower Reaches of Yangtze River), Ministry of Agriculture and Rural \\ affairs, Huazhong Agricultural University, Wuhan 430070, China; sheliangwang2017@mail.hzau.edu.cn (S.W.); \\ hao.zhang@webmail.hzau.edu.cn (H.Z.); leish@mail.hzau.edu.cn (L.S.); fangsenxu@mail.hzau.edu.cn (F.X.) \\ * Correspondence: dgd@mail.hzau.edu.cn
}

Received: 20 April 2020; Accepted: 20 May 2020; Published: 22 May 2020

\begin{abstract}
Phosphorus $(\mathrm{P})$ is an essential macronutrient required for plant growth and development. The involvement of cytokinin response factors $(\mathrm{CRFs})$ in phosphate $(\mathrm{Pi})$ homeostasis and lateral root (LR) initiation in Arabidopsis has been revealed. However, little is known in oil crops. Here, we performed genome-wide dissection of the CRF family in Brassica napus to identify 44 members, which were evolutionally classified into 6 subgroups. Among them, four BnaCRF8 genes were strongly upregulated by $\mathrm{P}$ deprivation, and were selected to be further investigated. Time course qRT-PCR analyses showed that four BnaCRF8 genes were enhanced dramatically after $12 \mathrm{~h}$ of $\mathrm{P}$ stress. Analyses of the subcellular localization in tobacco leaves indicated that BnaA7.CRF8 and BnaC2.CRF8 were localized in the nucleus. The expression of BnaCRF8 genes had constant negative effects on primary root growth and LR initiation and growth, and it reduced Pi acquisition and plant growth in Arabidopsis. Moreover, the expression of Pi homeostasis-related genes was modulated in BnaA7.CRF8 overexpression plants. These results suggest that BnaCRF8 genes might negatively regulate root architecture and plant growth through transcriptional modification of $\mathrm{Pi}$ homeostasis-related components. Overall, this study suggests that upregulation of BnaCRF8 genes might be a smart adaptive strategy to cope with continuous Pi deficiency in the environment.
\end{abstract}

Keywords: Brassica napus; cytokinin response factor; expression profile; CRF8; root architecture; Pi homeostasis; Arabidopsis; Nicotiana

\section{Introduction}

Phosphorus (P) is an essential macronutrient required for plant growth and development. P-containing molecules, such as nucleic acids, phospholipids, and ATP, participate in many physiological and metabolic processes, including signal transduction and the regulation of protein phosphorylation [1]. The bioavailability of utilizable inorganic phosphate (Pi) in soil usually cannot satisfy the demand of plants for their optimal growth [2]. Thus, a series of Pi starvation responses (PSRs), such as changes in the root morphology and architecture, induction or enhancement in the expression of high-affinity Pi transporters, changes in lipid modification, and exudation of organic acids and phosphatases into the soil, have evolved to adapt to Pi stress [3,4]. Pi limitation has been shown to upregulate the expression of two PHOSPHATE TRANSPORTER1 (PHT1) genes PHT1;1 and $P H T 1 ; 4$, and cause the accumulation of their proteins on the plasma membrane to facilitate Pi uptake in plants [5]. MiR399s are upregulated by Pi stress to reduce PHOSPHATE2 (PHO2) gene 
expression, resulting in the stability of PHOSPHATE1 (PHO1), which is responsible for Pi loading into the xylem in roots and translocation to the shoots [6-8]. PHO2, encoding the ubiquitin-conjugating E2 enzyme 24; interacts with PHT1;1, PHT1;4, and PHO1 proteins; and mediates their ubiquitination for degradation in cells [8]. Under Pi stress conditions, in order to reduce the demand for $\mathrm{P}$ in lipid metabolism, the concentration of phospholipids decreases and that of P-free lipid components, such as galactolipid, sulfoquinovosyldiacylglycerol (SQDG), and digalactosyldiacylglycerol (DGDG), increases [3]. Therefore, the lipid remodeling-related genes, including SQD1 and SQD2 (encoding enzymes in sulfolipid biosynthesis), $P L D \zeta 1$ and $P L D \zeta 2$ (encoding enzymes in phosphatidylcholine hydrolysis), and DGD1 and DGD2 (encoding digalactosyldiacylglycerol synthases), are induced by Pi depletion [9-11]. Furthermore, the LOW PHOSPHATE ROOT1/2 (LPR1/2) genes, which encode multicopper oxidases, have been identified as important regulators of meristem responses to low $\mathrm{Pi}$ availability in the primary root (PR) of Arabidopsis [12], while the PHOSPHATE ROOT DEVELOPMENT $(P R D)$ gene is involved in the regulation of root architectural responses to Pi starvation by controlling $\mathrm{PR}$ and lateral root (LR) elongation [13]. Moreover, many Pi starvation responsive transcription factors (TFs), such as PHOSPHATE STARVATION RESPONSE1 (PHR1) and PHR1-Like1 (PHL1), have been identified and shown to positively or negatively regulate several subsets involved in Pi homeostasis, as well as in root architecture modulation $[3,14,15]$.

The plant hormone cytokinin regulates numerous growth and developmental processes. The cytokinin response factors (CRFs) have been identified as cytokinin-regulated TFs [16]. CRFs belong to the B-5 subgroup of the ethylene response factor (ERF) subfamily of the APETALA2/ETHYLENE RESPONSIVE FACTOR (AP2/ERF) TF superfamily [17]. AP2/ERF proteins comprise one of the largest TF families in plants and are defined by the presence of an AP2 DNA binding domain of around 68 amino acids [18]. A total of $12 C R F$ genes in Arabidopsis have been identified depending on a clade-specific approximately 65 -amino acid CRF domain in the N-terminal region $[16,18]$. Among them, the CRF8 gene is induced specifically in Pi-deprived Arabidopsis roots and shoots, and a knockout mutant of the CRF8 gene phenotypically augments LR development, with higher Pi accumulation in plants [19]. In contrast, overexpression (OE) of AtCRF8 in Arabidopsis reduces both the PR length and LR initiation [19]. Besides AtCRF8, another two CRF genes (AtCRF2 and AtCRF3) are also involved in LR initiation and formation [20], while AtCRF1, AtCRF2, and AtCRF5 affect the development of the cotyledon, leaf, and embryo [16].

Allotetraploid rapeseed (Brassica napus, AACC, $2 \mathrm{n}=38$ ), which is formed by natural hybridization between $B$. rapa (AA, $2 n=20)$ and B. oleracea $(C C, 2 n=18)$ [21], is widely cultivated and has become the world's second leading crop source of vegetable oil (following soybean) for human consumption. Although the CRF genes in Brassica crops, including B. napus, have been identified and their evolutionary relationship has been discussed [22], the Pi homeostasis-related CRF genes in B. napus have not been assessed. Furthermore, their molecular function in plant $P i$ homeostasis remains unknown. In this study, we identified $44 C R F$ genes, including 4 novel genes in the B. napus genome, and estimated their transcriptional responses in Pi stress. The four low Pi-responsive BnaCRF8 genes were further investigated for their involvement in Pi homeostasis using OE transgenic Arabidopsis lines. Our work provides a comprehensive insight into the effects of BnaCRF genes on Pi homeostasis.

\section{Results}

\subsection{Identification and Phylogenetic Relationships of the CRF Family in Brassica napus}

To obtain the CRF family TFs in B. napus, we used the CRF protein sequences identified in the Arabidopsis genome to perform a BLASTP search against the B. napus genome. Arabidopsis has 12 CRF genes, and accordingly a total of $44 C R F$ genes were identified in the whole genome of $B$. napus. Except AtCRF9, the orthologous number of each AtCRF varied from 2 to 6 in B. napus (Table S1). To investigate the phylogenetic relationship of these BnaCRF genes, 21 and $22 C R F$ genes were identified from B. rapa and B. oleracea, respectively. Based on these CRF amino acid sequences, we generated a phylogenetic 
tree using the neighbor-joining (NJ) method (Figure 1). All CRF genes were evolutionally classified into six subgroups in which $C R F 1$ and $C R F 2$ were in subgroup $1, C R F 3$ and CRF4 were in subgroup 2, CRF5 and CRF6 were in subgroup 3, CRF7 was in subgroup 4, CRF8 was in subgroup 5, and CRF9, CRF10, $C R F 11$, and CRF12 were in subgroup 6. Most of the BnaCRFs located in the $A$ and $C$ subgenomes were consistently inherited from $B$. rapa and B. oleracea, respectively. However, the homologues of BraA10.CRF3 disappeared in B. napus, and BnaC3.CRF5 and BnaA9.CRF6 were additionally duplicated after hybridization between B. rapa and B. oleracea. The genes of CRF1, CRF3, CRF4, CRF7, CRF8, CRF10, and CRF12 suffered duplication after speciation of Arabidopsis and Brassica crops. The gene duplication events of CRF2, CRF5, CRF6, and CRF11 in Brassica crops were either after or parallel to Arabidopsis formation. CRF10 and CRF12 resulted from the duplication of CRF11, then they in turn expanded independently in Brassica crops.

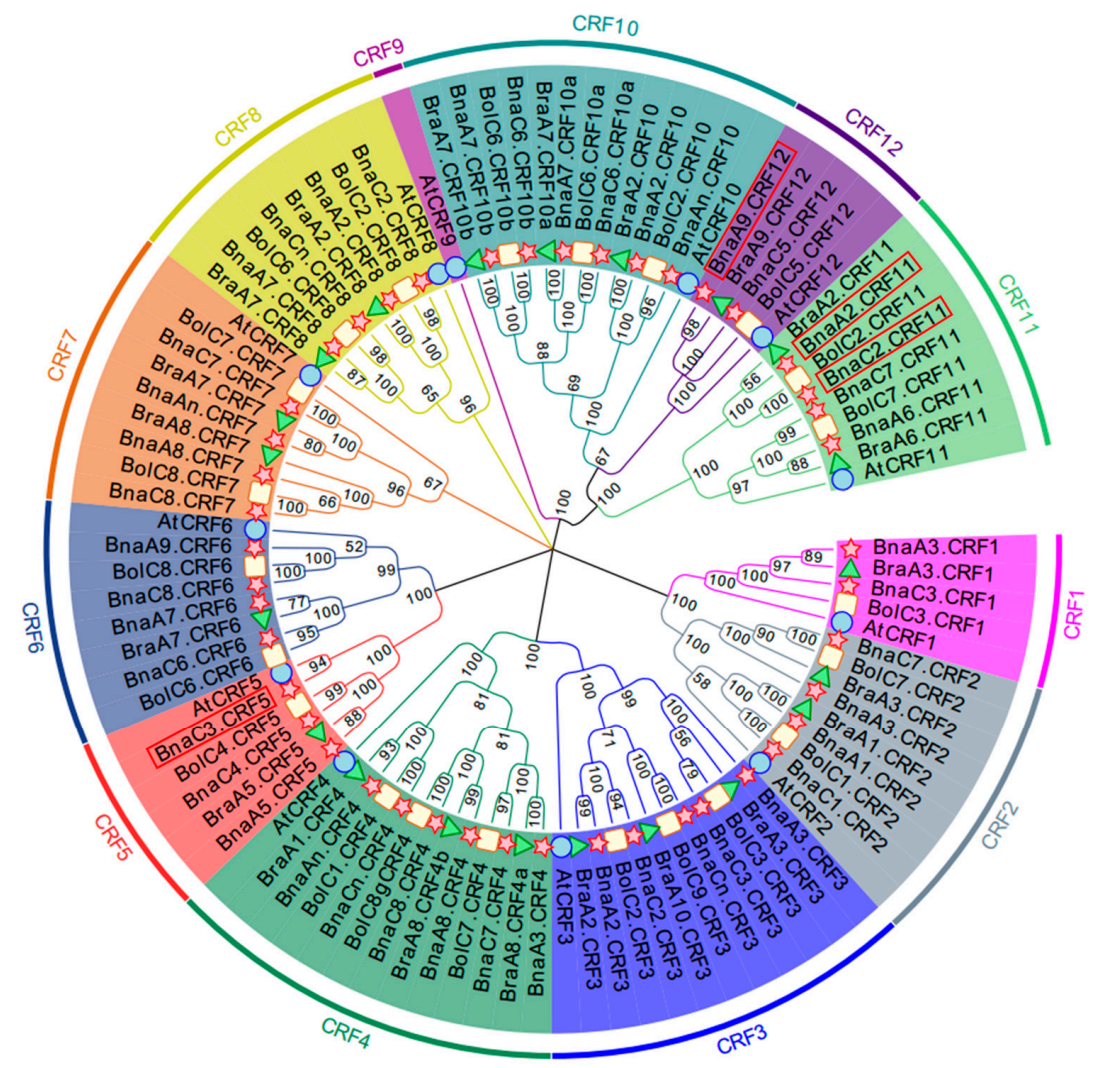

Figure 1. Phylogenetic tree of cytokinin response factor (CRF) genes in Arabidopsis thaliana and Brassica crops. Homologous genes of each CRF are distinguished by different colors. The unrooted tree was generated using ClustalW in MEGA7.0 using the full-length amino acid sequences of the 9, 44, 21, and 22 CRF proteins in A. thaliana (circle), B. napus (star), B. rapa (triangle), and B. oleracea (rectangle), respectively. Four BnaCRF genes newly identified in this research are highlighted with a red box.

\subsection{Gene Structure, Protein Features, and Chromosomal Duplication of the CRF Family in Brassica napus}

To analyze the gene structure of $B n a C R F s$, the genomic sequences identified above were schematically organized (Figure 2). The full length of the exons of all members ranged from 444 to 1569 bp (Table S1). Six BnaCRFs were identified with a single intron, in which the intron length of BnaC2.CRF11 was significantly longer than the others (Figure 2 and Table S1). BnaC3.CRF5 exclusively had two introns, although they were small. The remaining 37 BnaCRFs were identified consistently with one exon (Figure 2 and Table S1). The putative BnaCRF proteins varied in length from 147 to 351 amino acids (Figure 2 and Table S1). The deduced BnaCRF amino acid sequences were further analyzed for comprehensive prediction of the conserved motifs using the multiple expectation maximization for motif elicitation (MEME) suite [23]. A total of 10 conserved motifs were queried in 
BnaCRF proteins (Figure 2). Of these, motifs 1 and 2 found in all submitted members were AP2/ERF domains, while motif 3, representing the CRF domain, was present in 41 BnaCRF proteins but not in BnaA5.CRF5, BnaA2.CRF11, and BnaA9.CRF12 proteins (Figure 2 and Figure S1). In addition, we observed that evolutionally close CRFs displayed a similar composition of motifs although more or less different (Figures 1 and 2). The divergence in the composition of motifs and sequence length among BnaCRF proteins might determine their functional differentiation. It is important to note that both the AP2/ERF domain and CRF domain were conserved between B. napus and Arabidopsis (Figure S1). Further analyses of the physicochemical properties showed that the molecular weight (MW) of BnaCRF proteins ranged from 16.26 to $41.18 \mathrm{kDa}$, while the isoelectric point (PI) varied from 4.5 to 10 (Table S1). Chromosomal location analyses revealed that half of the CRF genes were distributed on the chromosomes of the A subgenome except A04 and A10. Similarly, the remaining 22 members were distributed on the chromosomes of the C subgenome except C04 and C09 (Table S1 and Figure S2). Furthermore, we identified 24 segmental duplication events but no tandem duplication events within the BnaCRF gene family by the BLASTP and MCScanX methods (Figure S3), indicating that segmental duplication events played a major driving force for the expansion of the CRF family in B. napus.
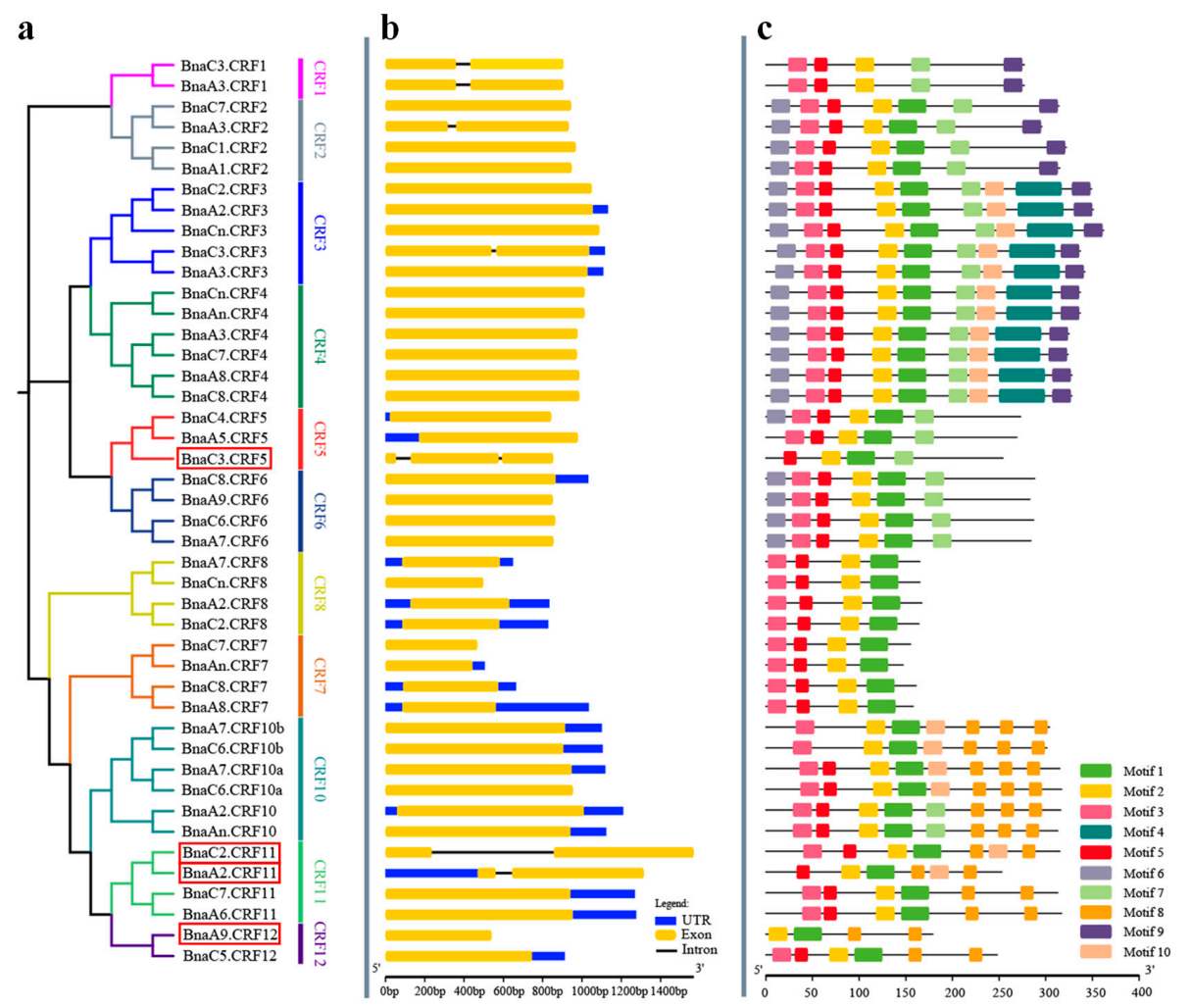

Figure 2. Phylogenetic relationships, gene structure, and architecture of conserved protein motifs in CRF genes from Brassica napus. (a) Phylogenetic relationships of BnaCRF genes. The phylogenetic tree (left panel) was constructed with MEGA 7.0 using the neighbor-joining (NJ) method with 1000 bootstrap replicates. Different homologous gene clusters are shown in different colors; (b) Gene structure of BnaCRF genes. Blue boxes indicate untranslated $5^{\prime}$ - and $3^{\prime}$-regions; yellow boxes indicate exons; black lines indicate introns. Scale bar represents gene length; (c) Motif characterization of BnaCRF proteins. The motifs, number 1-10, are displayed in different colored boxes. More sequence information for each motif is provided in Figure S1. The scale bar represents the protein length. Four BnaCRF genes newly identified in this research are highlighted with a red box. Motif 3 indicates the CRF domain, while motif 2 together with motif 1 indicate the APETALA2/ETHYLENE RESPONSIVE FACTOR (AP2/ERF) domain. 


\subsection{Expression Profiles of the BnaCRF Genes in Response to Phosphorus Stress}

Given the important roles of CRFs in nutrient stress regulation $[19,24]$, we tried to identify the critical $B n a C R F(s)$ in response to $\mathrm{P}$ stress. The expression profiles of the $44 \mathrm{BnaCRF}$ genes were investigated by the RNA-seq assay of the B. napus seedlings treated with or without $P$. The fragments per kilobase of exon per million mapped reads (FPKM) values of the BnaCRF genes were extracted from the RNA-seq data and were converted into logarithmic values shown by a heat map (Figure 3). In the roots, BnaC7.CRF4, BnaA9.CRF6, BnaC8.CRF6, BnaA2.CRF3, BnaC2.CRF3, and BnaA6.CRF11 were downregulated by P stress, while four BnaCRF8 (BnaA2.CRF8, BnaC2.CRF8, BnaA7.CRF8, and $B n a C n . C R F 8)$ genes were strongly upregulated by $P$ stress. In the leaves, the expression levels of five genes, including BnaA3.CRF2, BnaA3.CRF1, BnaA3.CRF3, BnaA7.CRF10a, and BnaC6.CRF10b, declined significantly under the low-P condition compared to that under the P-sufficient condition, while 11 genes were largely upregulated by $\mathrm{P}$ limitation, including the 4 BnaCRF 8 genes, which have the highest expression levels under $\mathrm{P}$ deprivation among all the CRF family members. To validate the expression profile of the four BnaCRF8 genes, we performed the qRT-PCR assay with treatments of $P$ deficiency for different times followed by P recovery (Figure 4). The results confirmed the RNA-seq data that the four BnaCRF8 genes were upregulated by $\mathrm{P}$ deficiency and had stronger expression levels in the leaves than in the roots under P deficiency. Taken together, these results imply that the four BnaCRF8 genes might participate in the growth regulation of plants in response to $P$ deficiency. Furthermore, pairwise alignment analyses showed that the amino acid identities of the four BnaCRF8 genes were more than $80 \%$ (Figure S4), implying their conserved roles in the P starvation response.

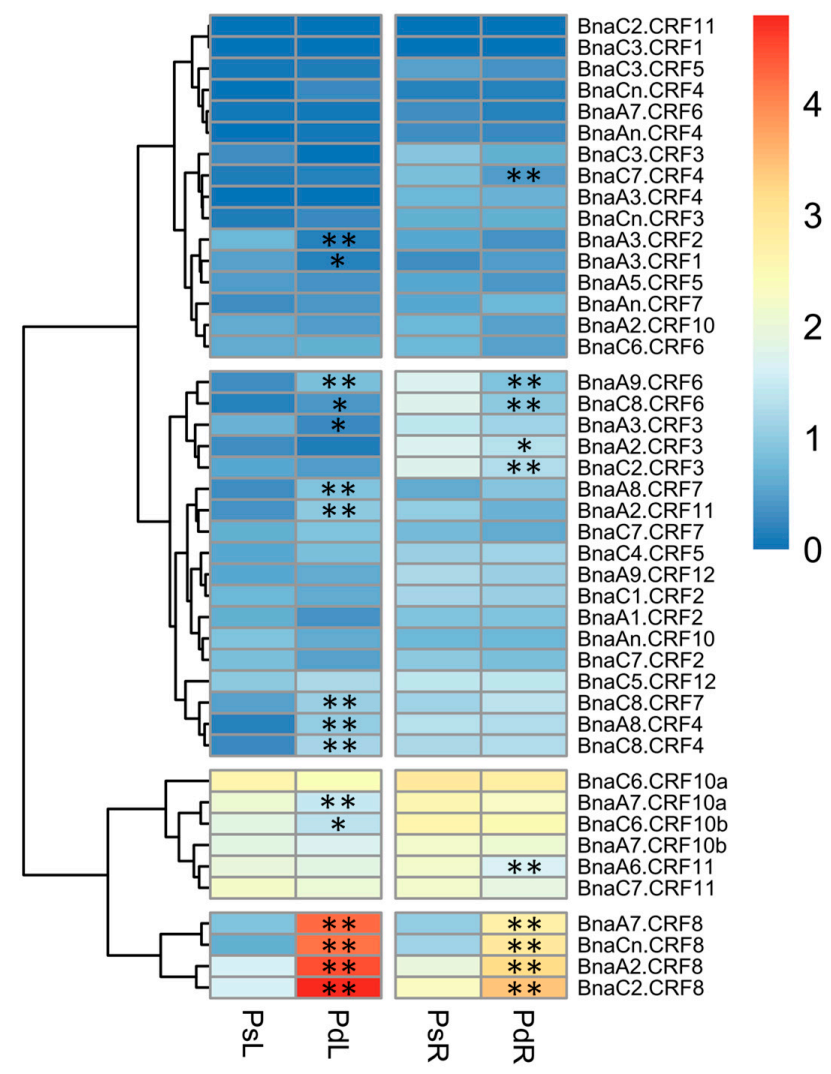

Figure 3. Expression profiles of the CRF gene family in response to phosphorus (P) stress in Brassica napus. Shoots and roots of P stress-treated plants were sampled for RNA-seq. The fragments per kilobase of exon per million mapped reads (FPKM) values of the 44 BnaCRFs were extracted and were converted into the logarithmic values. PsL: P-sufficient leaves; PdL: P-deficient leaves; PsR: P-sufficient roots; PdR: P-deficient roots. Asterisks indicate significant differences at ${ }^{*} p<0.05$ and ${ }^{* *} p<0.01$. 

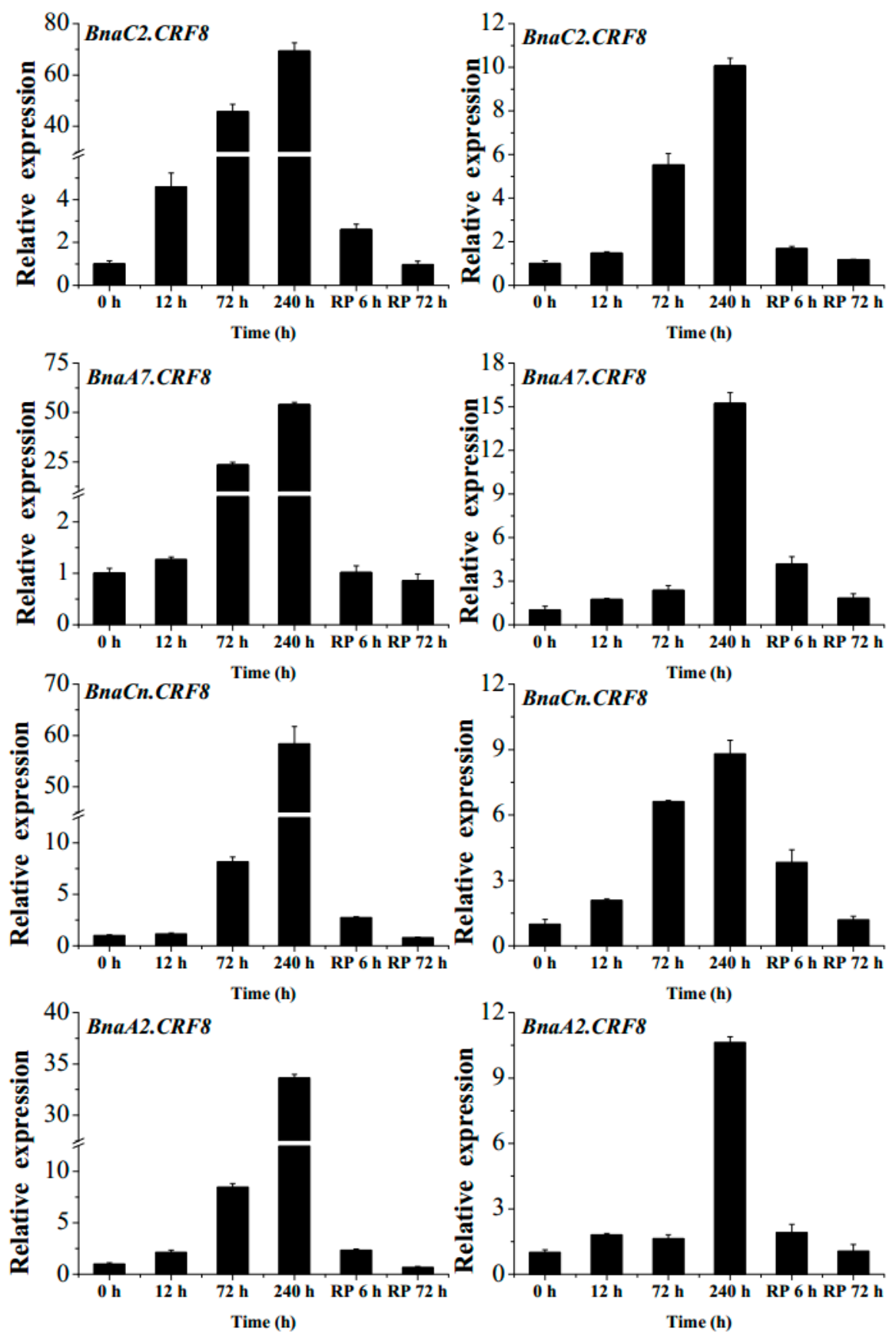

Shoot

Root

Figure 4. The expression changes of the 4 BnaCRF8 genes in response to phosphate (Pi) deficiency and recovery in Brassica napus. Fifteen-day-old plants were treated with Pi starvation $(0 \mu \mathrm{M} \mathrm{Pi})$ for 10 days before Pi recovery (RP). Then, the shoots and roots of the plants were sampled separately, and qRT-PCR was performed at 6 time points $(0,12,72$, and $240 \mathrm{~h}$ after Pi starvation, and 6 and $72 \mathrm{~h}$ after RP). Values represent mean \pm standard deviation (SD) of 3 biological replicates with 4 plants each.

\subsection{Subcellular Localization of the BnaCRF8 Genes}

In B. napus, we performed the subcellular localization analyses of BnaCRF proteins using the Wolf Psort program. The result showed that most of the BnaCRF proteins, including the four CRF8s, were predicted to be nuclear localized (Table S1). In line with this prediction, the CRF8 protein identified in Arabidopsis is localized in the nucleus as well [19]. To investigate the physiological roles of BnaCRF8 proteins in Pi homeostasis, BnaA7.CRF8 and BnaC2.CRF8 were further randomly selected 
to be experimentally examined for their subcellular localization in the plant cell. When transiently expressed in tobacco leaves, both BnaA7.CRF8-GFP and BnaC2.CRF8-GFP fusion protein signals were completely merged with the DAPI (4',6-diamidino-2-phenylindole, a blue-fluorescent DNA stain) signal (Figure 5). This result demonstrates that BnaCRF8 proteins localize in the nucleus.

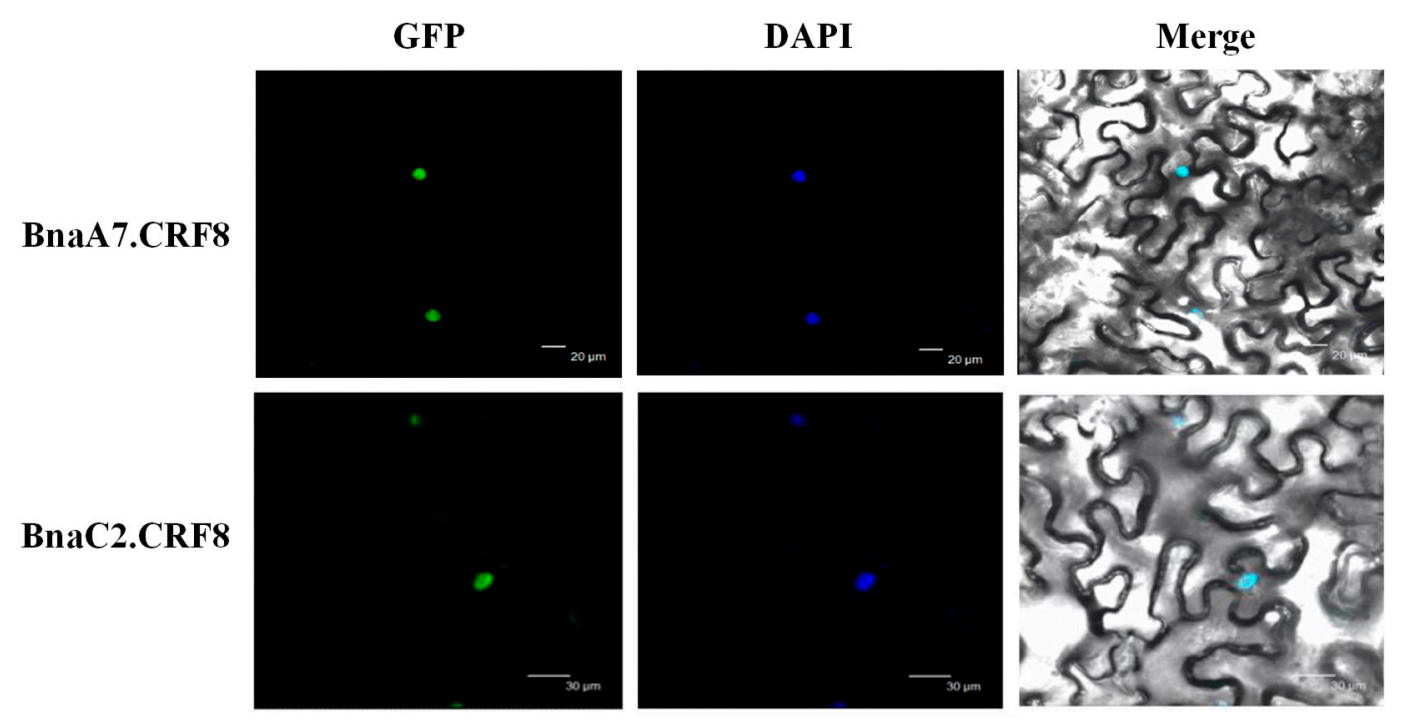

Figure 5. Subcellular localization of BnaA7.CRF8 and BnaC2.CRF8. Tobacco leaves were infiltrated with Agrobacterium harboring 35S::BnaA7.CRF8-GFP and 35S::BnaAC2.CRF8-GFP, respectively. DAPI (4',6-diamidino-2-phenylindole) staining was used to indicate the positions of nuclei. GFP, green fluorescent protein.

2.5. Phenotypical Characterization of the BnaCRF8 Overexpression Lines in Arabidopsis under Varied Phosphate Conditions

Given the nuclear localization and strong Pi-deficiency response of the BnaCRF8 genes, we further investigated their physiological roles in Pi homeostasis. To established transgenic lines of each BnaCRF8 in Arabidopsis, the 35S promoter was used to control their constant expression levels. Three independent T3 homozygous lines of each OE BnaCRF8 were selected to characterize the function in Pi homeostasis (Figure S5). PR and LR are two visible robust parameters of Pi homeostasis. Thus, the phenotypical parameters of 11-day-old seedlings grown in 10, 50, 625, and $1000 \mu \mathrm{M}$ Pi were analyzed and the results were analyzed statistically. OE of BnaA7.CRF8 in Arabidopsis developed shorter PR compared with the wild type (Col-0) regardless of the Pi concentrations (Figure 6a and Figure S6). The shorter PR was also observed in OE BnaC2.CRF8 lines at any Pi concentrations (Figure $6 \mathrm{~b}$ and Figure S7). Consistently, all OE BnaA2.CRF8 lines and OE BnaCn.CRF8 lines developed shorter PR compared with Col-0 plants at 50,625 , and $1000 \mu \mathrm{M} \mathrm{Pi}$, as well as some individual lines at $10 \mu \mathrm{M}$ Pi (Figure $6 c, \mathrm{~d}$ and Figure S7). Thus, these results suggest that $B n a C R F 8$ genes play a negative role in PR growth under varied Pi levels. 
a

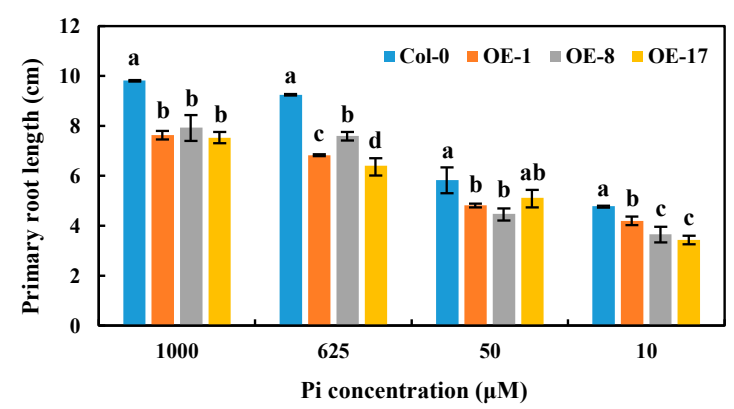

c

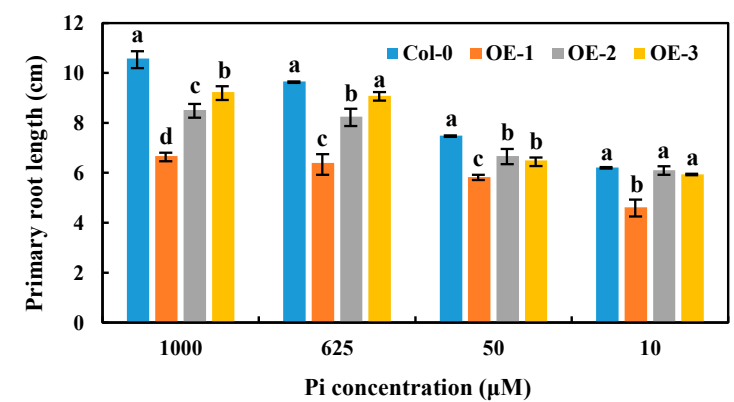

b

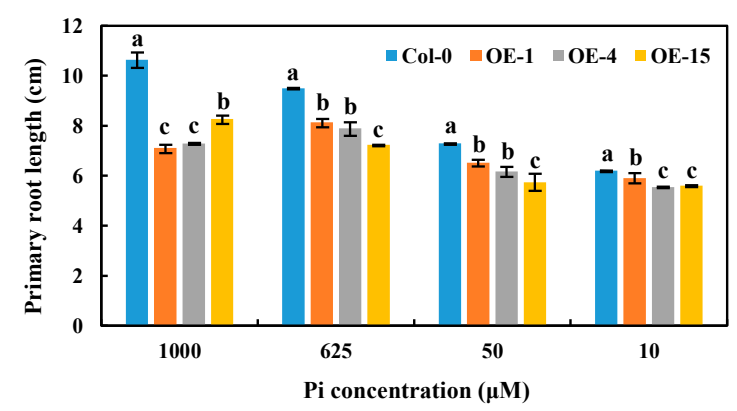

d

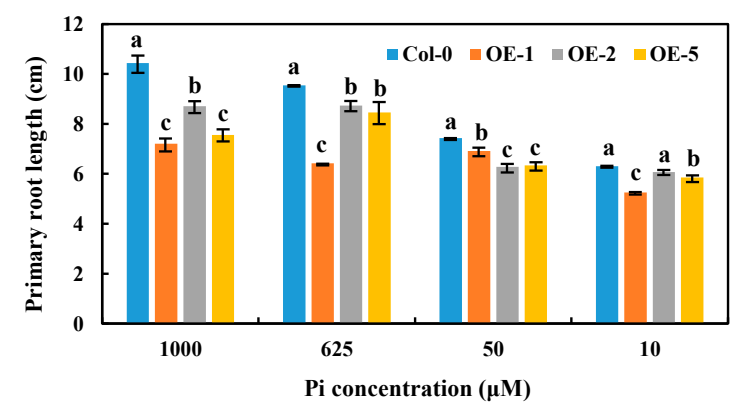

Figure 6. Primary root length comparison of the overexpression (OE) BnaCRF8 lines and the wild type (Col-0) at varied Pi conditions. Eleven-day-old plants grown on 10, 50, 625, and $1000 \mu \mathrm{M}$ Pi were imaged for primary root length measurement. (a) OE BnaA7.CRF8 lines; (b) OE BnaC2.CRF8 lines; (c) OE BnaA2.CRF8 lines; (d) OE BnaCn.CRF8 lines. Statistics were performed by Duncan's test at the significance level of $p<0.05$. Values represent mean $\pm \mathrm{SD}, \mathrm{n}=4$ replicates of 20 seedlings each. Small letters ( $\mathrm{a}, \mathrm{b}, \mathrm{c}$, and $\mathrm{d}$ ) above the bars indicate mean with significant differences at $p<0.05$ by Duncan's test.

High Pi concentrations (625 and $1000 \mu \mathrm{M})$ significantly disrupted the growth and reduced the number of LR in OE BnaA7.CRF8 lines compared with Col-0 plants. However, relatively low Pi concentrations (50 and $10 \mu \mathrm{M}$ ) had little effect on the growth and number of LR between OE BnaA7.CRF8 and Col-0 plants (Figure 7a,b). Interestingly, only the LR number was almost equal among OE BnaC2.CRF8 lines, OE BnaCn.CRF8 lines, and Col-0 plants at $50 \mu \mathrm{M} \mathrm{Pi}$, and these BnaC2.CRF8 lines and $B n a C n$.CRF8 lines had worse growth and a reduced number of LR relative to Col-0 plants at any Pi concentrations (Figure 7c-f). The constant negative regulation of the LR growth and number was observed in the OE BnaA2.CRF8 lines at all Pi concentrations tested (Figure 7g,h). These results suggest that OE of $B n a C R F 8$ genes negatively regulate the LR morphogenesis of the plants in response to the Pi dynamic. It is reasonable that the root function weakened by the expression of BnaCRF8 genes should hinder the shoot growth. With the exception of OE BnaCn.CRF8 at $10 \mu \mathrm{M} \mathrm{Pi}$, the fresh weights of the OE BnaCRF8 lines were totally lower than that of Col-0 at all Pi conditions tested (Figure S8). 
a

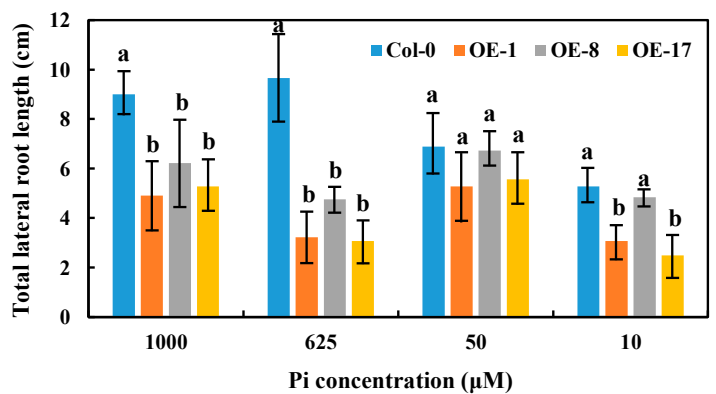

C

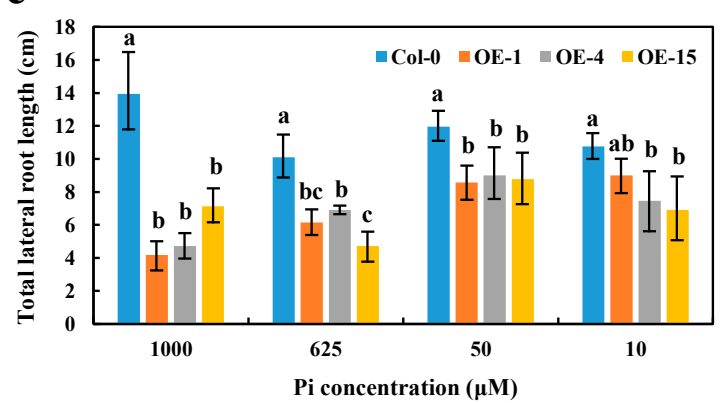

e

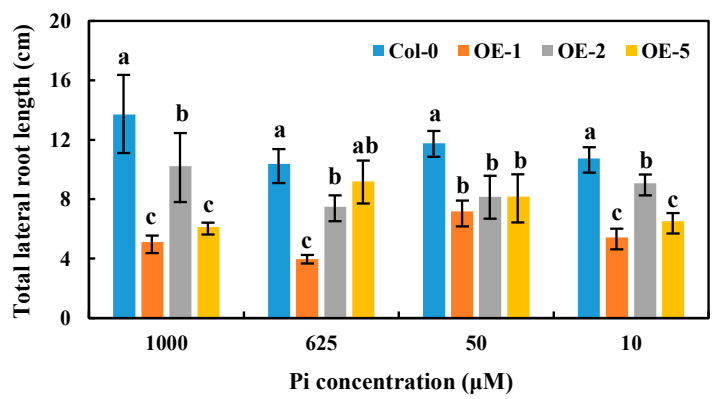

g

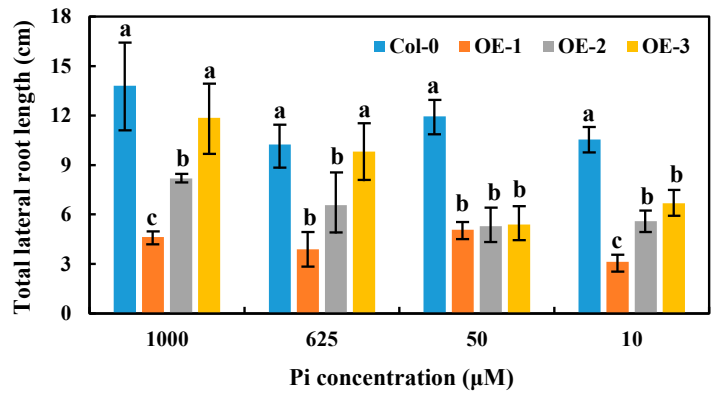

b

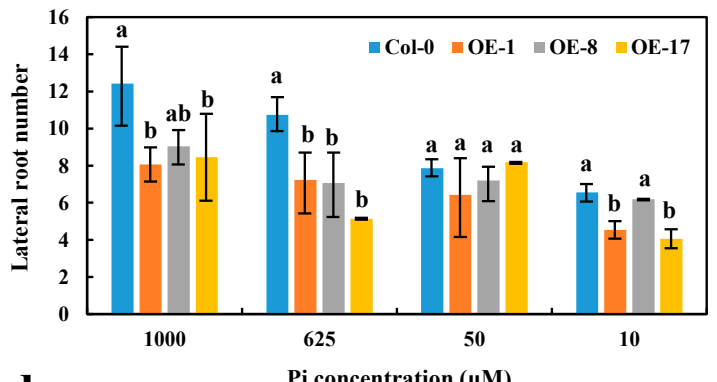

d

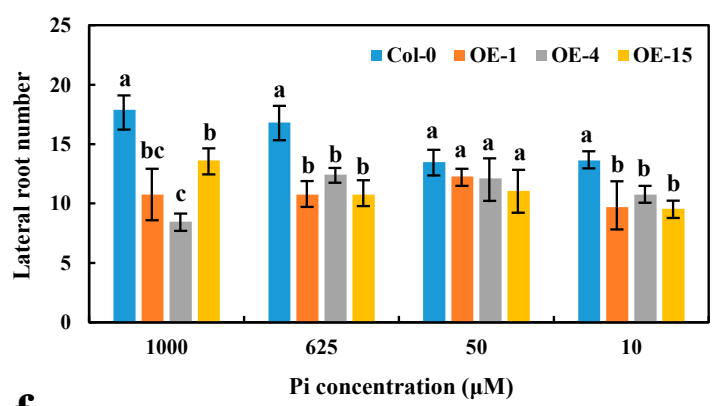

f

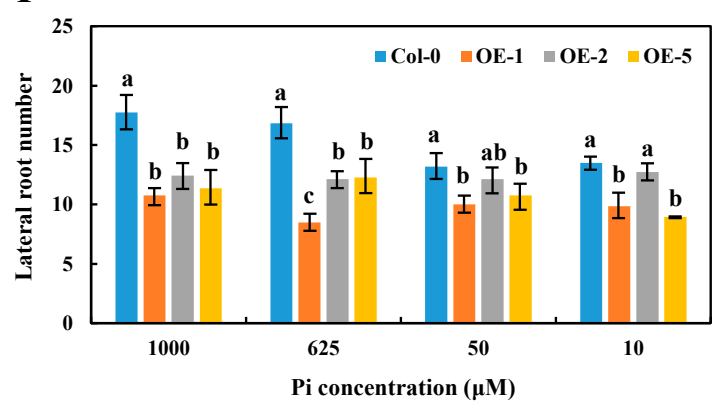

h

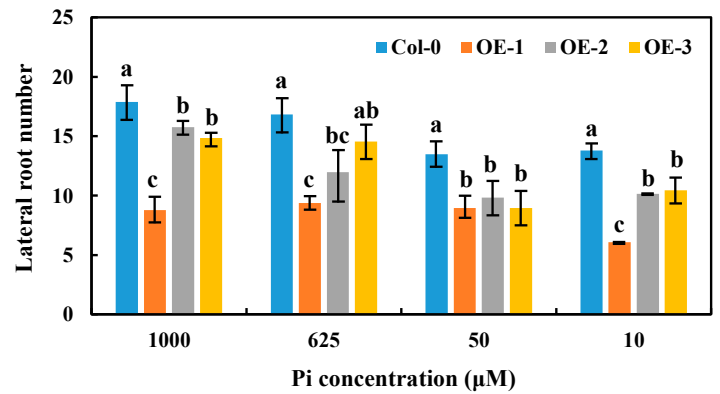

Figure 7. Comparison of the lateral root number and lateral root length among the OE BnaCRF8 lines and Col-0 at varied Pi conditions. Eleven-day-old plants grown on 10, 50, 625, and $1000 \mu \mathrm{M}$ Pi were imaged for measurement of the lateral root number and length. (a,b) OE BnaA7.CRF8 lines; (c,d) OE BnaC2.CRF8 lines; (e,f) OE BnaCn.CRF8 lines; (g,h) OE BnaA2.CRF8 lines. Statistics were performed by Duncan's test at the significance level of $p<0.05$. Values represent mean $\pm \mathrm{SD}, \mathrm{n}=4$ replicates of 20 seedlings each. Small letters $(a, b$, and $c)$ above the bars indicate mean with significant differences at $p<0.05$ by Duncan's test.

\subsection{BnaA7.CRF8 is a Negative Regulator of Phosphate Homeostasis in Arabidopsis}

It is evident that the expression of the BnaCRF8 genes limited root development to a larger extent at high Pi concentrations $(625$ and $1000 \mu \mathrm{M})$ than at low Pi concentrations (50 and $10 \mu \mathrm{M})$. We speculated that the Pi homeostasis underwent stronger disruption by BnaCRF8 gene expression at high Pi concentrations compared with low Pi concentrations. To this end, the OE BnaA7.CRF8 lines 
and Col-0 were used for further investigation at 1000 and $50 \mu \mathrm{M} \mathrm{Pi}$, which represent high-Pi and low-Pi treatments, respectively. There was a great reduction in the dry weight (DW) of OE BnaA7.CRF8 roots compared with Col- 0 roots at $50 \mu \mathrm{M} \mathrm{Pi}$, and it was even larger at $1000 \mu \mathrm{M} \mathrm{Pi} \mathrm{(Figure} \mathrm{8a).} \mathrm{The} \mathrm{Pi}$ concentrations of OE BnaA7.CRF8 lines were $81.8 \%-92.7 \%$ of Col-0 in roots and were $68.9 \%-93.3 \%$ of Col-0 in shoots at $50 \mu \mathrm{M}$ Pi (Figure 8b). The Pi concentrations of OE BnaA7.CRF8 lines were $68.8 \%-78.5 \%$ of Col-0 in roots and were $75.1 \%-80.9 \%$ of Col- 0 in shoots at $1000 \mu \mathrm{M} \mathrm{Pi}$ (Figure $8 \mathrm{~b}$ ). However, we could conclude that the changes in the Pi concentrations between the OE BnA7.CRF8 lines and Col-0 plants at $1000 \mu \mathrm{M}$ Pi were larger than that at $50 \mu \mathrm{M}$ Pi statistically. Moreover, the minimum concentration of $\mathrm{P}$ among all tested samples was $158 \mu \mathrm{M} \mathrm{g}^{-1} \mathrm{DW}$ in the roots of the \#17 OE BnaA7.CRF8 line at $50 \mu \mathrm{M} \mathrm{Pi}$ (Figure 8b), which is a sufficient $\mathrm{P}$ demand for plant growth. Thus, the Pi concentration reduction is not likely causal for the growth inhibition of the OE BnaA7.CRF8 lines. This is further supported by the comparable expression levels of Pi uptake transporters PHT1;1, PHT1;4, and PHO1 between Col-0 roots and OE BnaA7.CRF8 roots (Figure 9). Interestingly, the expression levels of LPR1/LPR2 and PRD were significantly higher and lower in OE BnaA7.CRF8 than in Col-0, respectively (Figure 9). LPR1/LPR2 trigger reactive oxygen species (ROS) to inhibit root tip growth under a low-Pi condition, and the $P R D$ gene is involved in the regulation of root architectural responses to Pi starvation by controlling PR and LR elongation (Table 1). In addition, the maintenance of Pi homeostasis requires components, such as membrane lipid remodeling factors $S Q D 1, P L D \zeta 2$, and the major regulator PHR1 (Table 1), all of which were suppressed in OE BnaA7.CRF8 roots relative to Col-0 roots (Figure 9). These results suggest that BnaA7.CRF8 might have a negative effect on Pi homeostasis without the direct regulation of Pi uptake in plants.

a

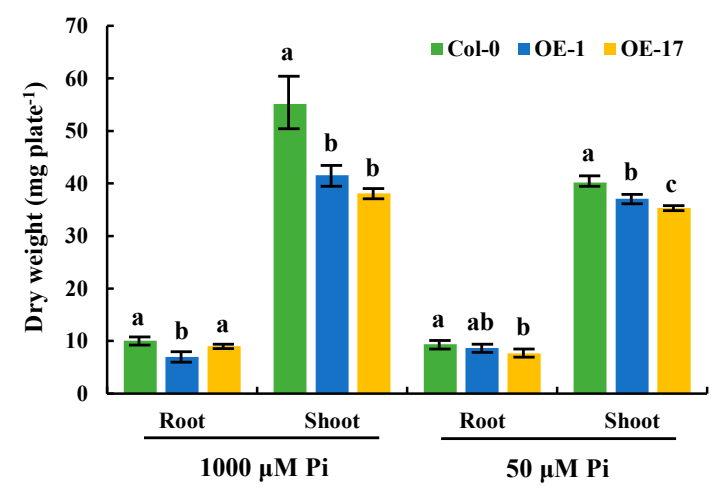

b

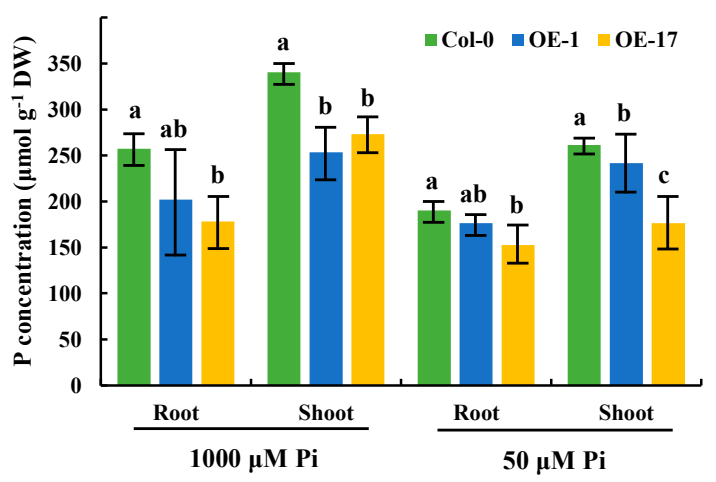

Figure 8. Effects of different phosphate (Pi) concentrations on the dry weight and endogenous Pi levels of the OE BnaA7.CRF8 lines and Col-0 plants. Eleven-day-old OE BnaA7.CRF8 lines and Col-0 plants grown on 50 and $1000 \mu \mathrm{M}$ Pi were imaged for measurement of the dry weight (a) and endogenous Pi levels (b). Values represent mean $\pm S D, n=4$ replicates of 20 seedlings each. Small letters $(a, b$, and c) above the bars indicate mean with significant differences at $p<0.05$ by Duncan's test.

Table 1. The molecular functions of the genes analyzed in this study.

\begin{tabular}{ccc}
\hline Gene Name & Molecular Function & References \\
\hline$P H T 1 ; 1$ & Pi uptake & {$[5]$} \\
$P H T 1 ; 4$ & Pi uptake & {$[5]$} \\
$P H O 1$ & Pi loading into xylem & {$[8]$} \\
SQD1 & Sulfolipid biosynthesis under Pi limitation & {$[9]$} \\
$P L D \zeta 2$ & Phosphatidylcholine hydrolysis and DGDG accumulation under Pi stress & {$[11]$} \\
LPR1/LPR2 & Root growth regulation under Pi stress & {$[12]$} \\
$P R D$ & Root growth regulation under Pi stress & {$[13]$} \\
$P H R 1$ & A central transcription factor involved in Pi starvation signaling & {$[14]$} \\
\hline
\end{tabular}
DGDG, digalactosyldiacylglycerol. 

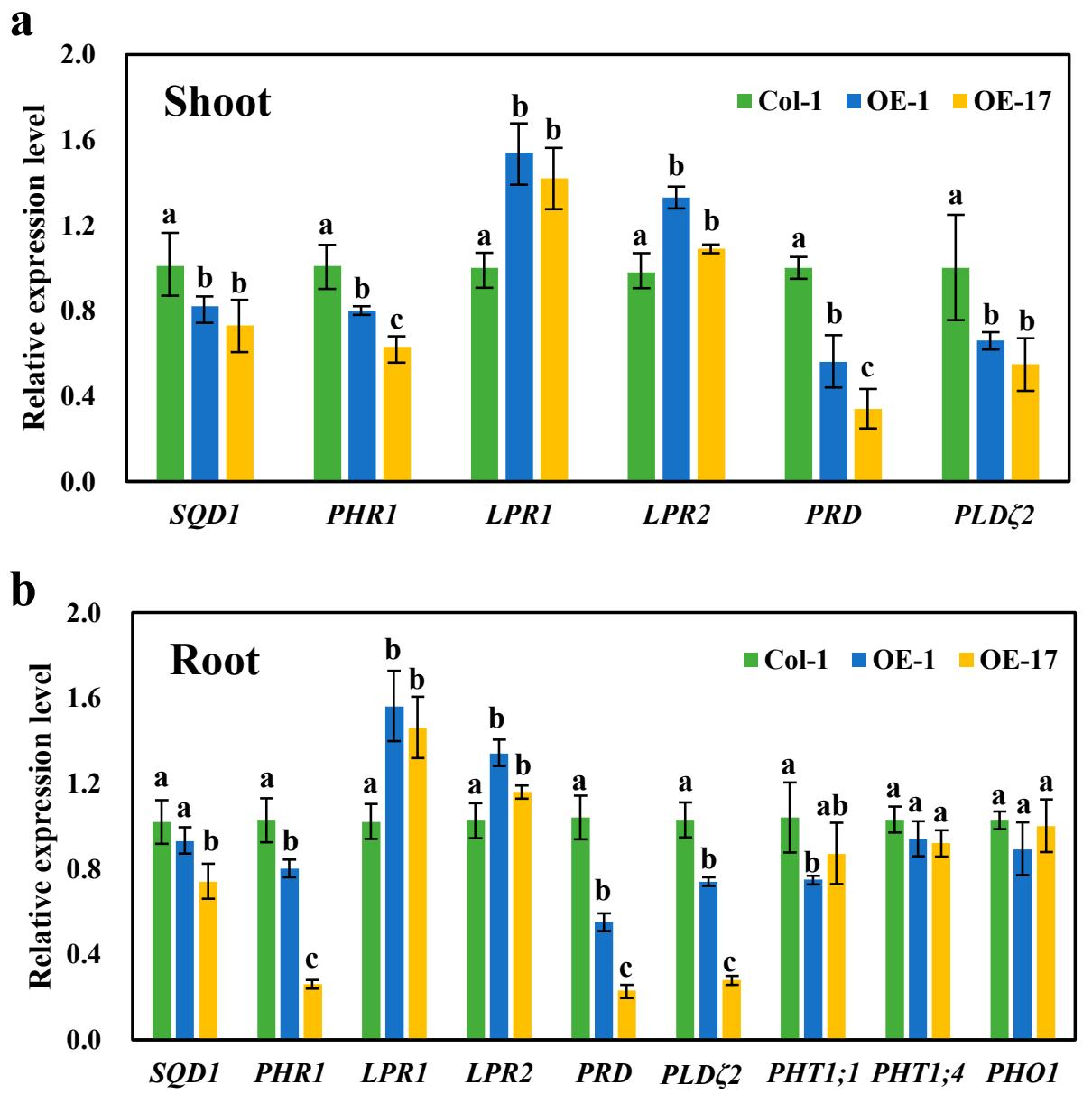

Figure 9. Relative expression levels of the Pi starvation-responsive genes in the wild-type (Col-0) and overexpression BnaA7.CRF8 lines. Shoots (a) and roots (b) of the 11-day-old plants grown on $1000 \mu \mathrm{M}$ Pi were used to perform qRT-PCR analyses. Data represent mean \pm SD of 3 biological replicates with 20 plants each. Different letters $(\mathrm{a}, \mathrm{b}$, and $\mathrm{c})$ indicate mean with significant differences at $p<0.05$ by Duncan's test. LPR1/2, LOW PHOSPHATE ROOT1/2; PHO1, PHOSPHATE1; PHR1, PHOSPHATE STARVATION RESPONSE1; PHT, PHOSPHATE TRANSPORTER; PRD, PHOSPHATE ROOT DEVELOPMENT; PLD 2 encodes an enzyme in phosphatidylcholine hydrolysis; $S Q D 1$ encodes an enzyme in sulfolipid biosynthesis.

\section{Discussion}

TFs are the most central regulator to transduce environmental stresses to activate adaptive gene networks in plants. Pi deficiency is a common abiotic stress in agricultural production, and has gained much research attention by botanists and biologists over the last decades in order to improve plant growth in response to Pi deprivation [2]. Based on the transcriptomic analyses, thousands of differentially expressed genes were identified, including many TFs, such as NAC (NAM, ATAF1/2, CUC2), MYB (v-myb avian myeloblastosis viral oncogene homolog), AP2/ERF, C2H2 (Cys2/His2 zinc finger protein), bHLH (basic helix-loop-helix protein), WRKY (pronounced 'worky', WRKY domain containing protein), and CCAAT-binding families [25-29]. The CRF family is a small subset of the AP2/ERF TF superfamily and was identified in Arabidopsis and other plants [18,30]. In B. napus, a total of $40 C R F$ genes were identified previously [22]. In this study, four novel CRF genes (Figure 1 and Table S1) were found based on the genome-wide dissection of B. napus using Arabidopsis CRF family information. Since the conserved CRF-specific motif was not evident in three novel BnaCRF proteins (Figure 2 and Figure S1), they have likely been ignored in previous identification using the CRF domain as a search query [22]. Brassica crops have a bigger biomass compared with Arabidopsis, and a 
sophisticated stress response system is always necessary to fine-tune their development and growth. The frequential expansion of genes through gene duplication, such as the greater number of $C R F$ genes in B. napus relative to Arabidopsis, is an efficient adaption strategy in an ever-changing environment.

Not all $C R F$ genes are cytokinin responsive, but a range of environmental stimuli, including biotic and abiotic stresses, are the cues [19,30-32]. In Arabidopsis, CRF genes play broader roles in reproductive development, embryogenesis, root architecture, and abiotic stress adaption [19,20,32-35]. For example, AtCRF4 is rapidly upregulated after exposure to high-nitrogen $(\mathrm{N})$ conditions, and OE of AtCRF4 largely disrupts $\mathrm{N}$ homeostasis and reduces growth in Arabidopsis [36], as AtCRF4 controls several key N-responsive TFs [24]. AtCRF8 is specially induced in Pi-deprived shoots and roots, and RNA interference of $A t C R F 8$ results in augmented LR and Pi accumulation in plants, while OE of AtCRF8 limits root growth [19]. In line with these results, all the CRF8 genes are strongly induced by Pi deficiency in B. napus leaves and roots (Figure 3), and OE BnaCRF8 lines tend to reduce PR growth, LR number and elongation, and Pi concentration from low-Pi to high-Pi conditions (Figures 6-8). The Pi concentration reduction in the OE BnaA7.CRF8 lines could not be ascribed to the Pi transporters because their transcription activities were not altered between OE BnaA7.CRF8 lines and Col-0 plants (Figure 9). Expression of AtPHR1 is constitutive under different Pi conditions and the Arabidopsis mutant phr1 showed impaired expression of most PSR genes and Pi allocation [14]. It is a possibility that the downregulation of PHR1 results in low Pi uptake in OE BnaA7.CRF8 lines (Figures 8 and 9). The limited growth of PR and LR by OE of BnaA7.CRF8 contributes to the decrease in root surface area, which probably downregulates the Pi uptake efficiency as well. Decreased expression of PHR1 in OE BnaA7.CRF8 lines presumably alters the expression profile of a series of PSR genes. Further investigation is required to assess the relationship between PHR1-mediated PSR genes and root architecture alternation in OE lines.

Expression of BnaA7.CRF8 modulated several PSR genes in the roots, leading to negative effects on Pi homeostasis (Figure 9). The Arabidopsis mutants lpr1, lpr2, and lpr1/lpr 2 are insensitive to low $\mathrm{Pi}$ stress with a longer PR length than that of the Col-0 plants [12], while OE of LPR1 reduces the PR length of Arabidopsis compared to the control plant [37]. It has been demonstrated that LPR1-meidated ROS production is essential for the inhibition of PR growth under Pi deficiency [37]. In OE BnaA7.CRF8 lines, the mRNA abundances of LPR1 and LPR2 were higher than that of Col-0 (Figure 9), suggesting that increased ROS might accumulate in the OE BnaA7.CRF8 lines. Arabidopsis with functional disruption of PRD has less-developed LR and PR under Pi deficiency, but the expression levels of PHT1;1 and PHT1;4 are similar to that of Col-0 plants [13]. In agreement with this, the expression levels of Pi transporter genes, including PHT1;1, PHT1;4, and PHO1, were similar between the OE BnaA7.CRF8 lines and Col-0 plants, and the expression of the PRD gene was lower in the OE BnaA7.CRF8 lines relative to Col-0 plants (Figure 9). This result suggests that the decreased expression of the PRD gene might inhibit root architecture development in the OE BnaA7.CRF8 lines. Another well-known adaptive mechanism to $P$ depletion in plants is lipid remodeling. Under Pi-stress conditions, plants can replace membrane phospholipids to P-free sulfolipids and galactolipids to save Pi for vital metabolisms [3]. Genes involved in these processes, such as $S Q D 1 / 2, D G D 1 / 2$, and $P L D \zeta 1 / 2$, are found to be upregulated by Pi starvation [3,9-11]. However, the expression levels of $S Q D 1$ and $P L D \zeta 2$ genes were inhibited by

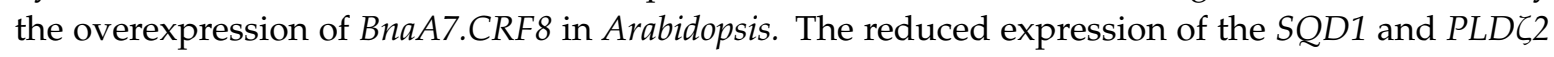
genes in the OE BnaA7.CRF8 lines indicates a low sulfolipid accumulation level and slow hydrolysis of phosphatidylcholine, which are obviously detrimental for Pi homeostasis. We noted that BnaCRF8 genes had a stronger low-Pi response in shoots than that in roots (Figure 4), and the DW of OE BnaA7.CRF8 lines was reduced significantly relative to Col-0 under 50 and $1000 \mu \mathrm{M}$ Pi (Figure 8). This implies that BnaCRF8 genes may play more important roles in Pi homeostasis in shoots than in roots. Except for BnaC2.CRF8 in the roots of B. napus, no more than 2-fold changes were detected for the other three genes within $12 \mathrm{~h}$ after low-Pi treatment (Figure 4), demonstrating that the transcriptional response of $B n a C R F 8$ genes to low Pi is not an early event compared to many other TFs that are rapidly induced by low Pi. Transcriptional activities of the BnaCRF8 genes were intensified upon prolonged 
Pi-deficiency treatment and were reversibly downregulated after Pi resupply (Figure 4), suggesting that BnaCRF8 genes mainly participate in the adaption response to Pi deficiency at a late stage. It has been believed that the long-term Pi deficiency certainly retarded plant growth and development. Therefore, there must be an efficient strategy employed by plants to cope with continuous Pi stress and to regulate plant growth. Based on the findings in this study, we propose that BnaCRF8 genes are one kind of central TF genes regulating plant growth and development under continuous Pi deficiency.

Taken together, we identified $44 C R F$ genes, including 4 novel genes, on the whole genome of B. napus, and found 4 BnaCRF8 genes that are strongly upregulated by low Pi stress. Phenotypic characterization of OE BnaCRF8 Arabidopsis plants combined with the analyses of Pi homeostasis-related genes and Pi concentrations in plants suggest that the upregulation of BnaCRF8 genes by low-Pi stress might be a smart adaptive strategy to cope with continuous Pi deficiency in the environment.

\section{Materials and Methods}

\subsection{Identification of CRF TFs in the Brassica napus Genome}

CRF genes were identified in B. napus based on the 12CRF protein sequences from Arabidopsis $[16,18]$ using the BLASTP search program in the CNS-Genoscope database (http://www.genoscope.cns.fr/ brassicanapus/) [21], which provides gene annotation, including the chromosome localization and genomic sequence. Redundant sequences were removed manually. The CRF domain and AP2/ERF domain of all identified BnaCRF genes were future analyzed using the SMART database (http://smart. embl-heidelberg.de/) [38], and NCBIConserved Domain Search database (http://www.ncbi.nlm.nih.gov/ Structure/cdd/wrpsb.cgi) [39]. The physicochemical parameters of BnaCRF proteins, including MW and PI, were calculated using the ExPASy tool (http://www.expasy.org/tools/) [40]. Subcellular localization prediction was conducted using Wolf Psort (http://www.genscript.com/wolf-psort.html) [41].

\subsection{Phylogenetic and Conserved Motif Analyses of BnaCRF Genes}

Multiple alignments of candidate CRF protein sequences were carried out using ClustalW with default parameters [42]. An unrooted phylogenetic tree of all CRF proteins from B. napus, B. rapa, B. oleracea, and Arabidopsis were generated with MEGA6 [43] using the NJ method with the following parameters: Poisson correction, complete deletion, and 1000 bootstrap replicates. Conserved motifs in B. napus were identified using the motif-finding tool MEME (Multiple EM for Motif Elicitation, $\mathrm{V}$ 4.11.2) [23]. MEME searching was performed across BnaCRF protein sequences using the following parameters: (1) Optimum motif width was set to $\geq 6$ and $\leq 100$; (2) the maximum number of motifs was set to identify 10 motifs; and (3) the others were the default settings.

\subsection{Chromosomal Location and Gene Duplication Analyses}

Physical location information of the BnaCRF genes was retrieved from the CNS-Genoscope genome database, and was mapped to rapeseed chromosomes using Circos [44]. Gene duplication events and collinearity relationships were analyzed using the Multiple Collinearity Scan toolkit (MCScanX) [45]. The criteria for analyzing potential gene duplications were: (a) Length of alignable sequence covers $>75 \%$ of the longer gene, and (b) similarity of aligned regions $>75 \%$.

\subsection{Plant Materials and Treatments}

The P-efficient B. napus genotype 'Eyou Changjia' [46] and Arabidopsis Col-0 ecotype were used in this study. Plants were cultured in a hydroponic condition in an illuminated culture room with a cycle of $16 \mathrm{~h} / 24^{\circ} \mathrm{C}$ day and $8 \mathrm{~h} / 22^{\circ} \mathrm{C}$ night, and a light intensity of 300-320 $\mu \mathrm{mol}$ proton $\mathrm{m}^{-2} \mathrm{~s}^{-1}$. The modified Hoagland's solution was used for plant growth [47]. The surface-sterilized seeds were immersed for $24 \mathrm{~h}$ in deionized water prior to germination on moistened gauze. Six-day-old seedlings were transferred into a 10-L black plastic container filled with quarter-strength nutrient solution for 5 day's growth followed by half strength and full strength with an interval of 5 days. Seedlings were 
fixed on a perforated foam board with sponge. For the phenotypic characterization, Arabidopsis were grown vertically on solid medium $(1.5 \%(w / v)$ sucrose and $0.9 \%(w / v)$ agar with $10,50,625$, and $1000 \mu \mathrm{M}$ Pi in Petri dishes).

For RNA-seq analyses in B. napus, 15-day-old seedlings were transferred to nutrient solutions with $0 \mathrm{mM}$ or $250 \mu \mathrm{M}$ Pi for 10 days. The leaves and roots were harvested for RNA extraction, respectively. For the qRT-PCR assay in B. napus, 15-day-old seedlings were treated with P-free nutrient solutions. Samples were harvested at $0,12,72$, and $240 \mathrm{~h}$ after P-free treatment, respectively. After 10 days' treatment, Pi recovery $\left(250 \mu \mathrm{M} \mathrm{KH}_{2} \mathrm{PO}_{4}\right)$ was added to the nutrient solution for 6 and $72 \mathrm{~h}$. Three biological replicates with 4 plants each were included. For the qRT-PCR assay in Arabidopsis, the shoots and roots of the 11-day-old plants grown on $1000 \mu \mathrm{M}$ Pi were harvested for RNA extraction. Three biological replicates with 20 plants each were included. All samples were stored at $-80{ }^{\circ} \mathrm{C}$ prior to RNA extraction.

\subsection{RNA Isolation and Real-Time Quantitative PCR}

Total RNA was isolated using the total RNA kit (Invitrogen, Carlsbad, CA, USA) according to the manufacturer's instructions. First-strand cDNA was synthesized using the Prime Script RT reagent Kit (Promega, Madison, WI, USA). Real-time RT-PCR was performed using ABI7300 Real-time Detection System (Applied Biosystems, Foster City, CA, USA) with SYBR Premix Ex TaqTM II (Toyobo, Shanghai, China). The PCR conditions were set as follows: $95^{\circ} \mathrm{C}$ for $2 \mathrm{~min} ; 40$ cycles of $95^{\circ} \mathrm{C}$ for $30 \mathrm{~s}$ and $60^{\circ} \mathrm{C}$ for $30 \mathrm{~s}$; and $72{ }^{\circ} \mathrm{C}$ for $1 \mathrm{~min}$. The $2^{-\Delta \Delta C T}$ method was used to calculate the relative expression levels of BnaCRF8 genes using Actin as the reference gene. The primers of the 4 BnaCRF8 genes and the Actin gene used for qRT-PCR detection are listed in Table S2.

\subsection{Expression Pattern Analysis of BnaCRF Genes in Response to Pi Starvation using RNA-Seq Data}

The fully expanded leaves and roots of seedlings treated with $0 \mathrm{mM}$ or $250 \mu \mathrm{M}$ Pi were harvested separately for RNA extraction using TRIzol reagent according to the manufacturer's instructions (Invitrogen, Carlsbad, CA, USA). Three biological replicates with 4 plants each were included. The quality and integrity of the total RNA were assessed using a NanoDrop 2000 spectrophotometer (Thermo Fisher Scientific, Waltham, MA, USA). Then, 12 RNA samples were subjected to an Illumina Hiseq 2000 platform (Illumina, San Diego, CA, USA) with 100-bp paired-end reads. Finally, a total of $643,846,484$ raw reads and $571,929,682$ clean reads were generated with an average of $\sim 5.0 \mathrm{~Gb}$ sequencing data for each sample. Sequencing reads were aligned to the $B$. napus reference genome [21] with bowtie v2.0.6 and then assembled using TopHat v2.0.12 [48]. The mapped reads were 496,073,698 and the unique mapped reads were 430,982,901 (75.40\%). The transcript abundance of each gene was estimated using FPKM based on the length of the gene and reads count mapped to this gene. Differently expressed genes between $+\mathrm{P}(250 \mu \mathrm{M} \mathrm{Pi})$ and $-\mathrm{P}(0 \mathrm{mM} \mathrm{Pi})$ treatments were filtered using the Benjamini and Hochberg's approach by controlling the false discovery rate (FDR) of 0.05 .

\subsection{Vector Construction and Arabidopsis Transformation}

All PCR amplifications of the 4 BnaCRF8 genes were conducted using Phusion Polymerase (New England Biolabs (Beijing) Ltd., Beijing, China) with the specific primers (Table S2). The PCR reaction product was digested and ligated into EcoRI/XhoI sites of the binary vector pBin35SRed to generate a 35S::BnaCRF8 construct. Arabidopsis Col-0 grown on vermiculite to the bud stage was used, and genetically transformed Arabidopsis were produced using the Agrobacterium tumefaciens-mediated method [49]. The T3 homozygous generation was established due to the DsRed fluorescence observation [50].

\subsection{Transient Expression Assays in Nicotiana benthamiana}

To construct 35S::BnaA7.CRF8-GFP and 35S::BnaA2.CRF8-GFP, the open reading frame sequences of BnaA7.CRF8 and BnaA2.CRF8 were fused in frame into the position between the $35 \mathrm{~S}$ promoter and 
green fluorescent protein (GFP) sequence in pMDC83 plasmids. An Agrobacterium carrying GV3101 construct was harvested by centrifugation and suspended in the solutions containing $10 \mathrm{mM}$ MES, $\mathrm{pH} 5.6,10 \mathrm{mM} \mathrm{MgCl} 2$, and $200 \mathrm{mM}$ acetosyringone to an optical density $(600 \mathrm{~nm})$ of 0.8 , incubated at room temperature for $3 \mathrm{~h}$, and then used to infiltrate the leaves of tobacco (Nicotiana benthamiana) using a needle-free syringe. Nicotiana benthamiana was grown in a chamber with a $14 \mathrm{~h} / 25^{\circ} \mathrm{C}$ day and $10 \mathrm{~h} / 20^{\circ} \mathrm{C}$ night cycle, and a light intensity of $\sim 200 \mu \mathrm{mol}$ proton $\mathrm{m}^{-2} \mathrm{~s}^{-1}$. After 2 days' growth, the cellular localization of BnaCRF8-GFP protein in the infiltrated leaves was checked using a Leica SP8 lazer confocal microscopy system (Leica Microsystem, Wetzlar, Germany). DAPI was used to stain the nucleus. The wavelength sets were GFP (excitation: $488 \mathrm{~nm}$, emission: 505-545 nm), DAPI (excitation: $405 \mathrm{~nm}$, emission: 415-450 nm). The images were recorded by the Leica SP8 system and data were further analyzed using ImageJ software (http://rsb.info.nih.gov/ij/).

\subsection{Measurements of Root Traits}

After harvesting, the roots of Arabidopsis were scanned at $600 \mathrm{dpi}$ with a scanner. The different root traits, including PR length, LR number, LR length, and total root length, were evaluated using the root image analyses software WinRHIZO Pro (Regent Instruments, Montreal, QC, Canada). Four replicates with 20 plants each were analyzed.

\subsection{Quantification of Total Phosphorus}

Leaves and roots of 11-day-old Arabidopsis plants treated with different Pi concentrations were sampled, and then dried at $65^{\circ} \mathrm{C}$ to a constant weight. Then, the samples were pre-digested in glass tubes with $\mathrm{H}_{2} \mathrm{SO}_{4}$ for $12 \mathrm{~h}$ followed by $\mathrm{H}_{2} \mathrm{O}_{2}$ addition at $180{ }^{\circ} \mathrm{C}$ until the solution became colorless. The digestion was continued for another $30 \mathrm{~min}$. The Pi concentration in the samples was quantified as described previously [7]. Four replicates with 20 plants each were analyzed.

\subsection{Statistical Analyses}

Statistics were performed by Duncan's test at the significance level of $p<0.05$.

Supplementary Materials: Supplementary materials can be found at http://www.mdpi.com/1422-0067/21/10/3660/ s1, Figure S1: Conserved domain analyses of CRF genes in Brassica napus. a, Sequence alignment of conserved CRF domain in B. napus and Arabidopsis thaliana; $\mathbf{b}$, Sequence alignment of conserved the AP2/ERF domain in $B$. napus and $A$. thaliana; $\mathbf{c}$, The sequence information of the CRF domain and AP2/ERF domain; $\mathbf{d}$, The sequence information of 10 motifs in Figure 2, Figure S2: Chromosomal localization of 44 CRF genes in Brassica napus genome. Chromosome name is written as A01-A10 and C01-C09. The size of each chromosome was estimated as $\mathrm{Mb}$ on the bottom of each chromosome. Bar $=10 \mathrm{Mb}$, Figure S3: Gene duplication of BnaCRF genes in Brassica napus genome. The genome visualization tool CIRCOS was used to illustrate the chromosomal distribution of $B n a C R F$ genes. The chromosome number is shown inside the circle. The chromosomal location of each BnaCRF is shown outside of the corresponding chromosome. Duplicated gene pairs are linked by green lines, Figure S4: Pairwise identity matrix of CRF8 subfamily proteins. CRF8 proteins in Arabidopsis thaliana (At), Brassica napus (Bna), B. rapa (Bra) and B. oleracea (Bol) were used for pairwise alignment. The amino acid identities $(\%)$ are indicated by the number and column, Figure S5: Semi-qRT-PCR analyses of the expression levels of 4 CRF8 genes in Col-0 and transgenic lines. Actin was used as the internal control. All the experiments were repeated twice with identical results, Figure S6: Phenotypical performances of the 11-day-old wild-type (Col-0) and BnaA7.CRF8 gene overexpression (OE) lines under $1000 \mu \mathrm{M}, 625 \mu \mathrm{M}, 50 \mu \mathrm{M}$ and $10 \mu \mathrm{M}$ Pi. OE lines and Col-0 plants were separated by red lines, Figure S7: Phenotypical performances of the 11-day-old wild-type (Col-0) and overexpression (OE) lines (BnaC2.CRF8, BnaCn.CRF8 and BnaA2.CRF8) under $1000 \mu \mathrm{M}, 625 \mu \mathrm{M}, 50 \mu \mathrm{M}$ and $10 \mu \mathrm{M}$ Pi. OE lines and Col-0 plants were separated by red lines, Figure S8: Fresh weights of the 11-day-old wild-type (Col-0) and 4 BnaCRF8 genes overexpression lines under different Pi concentrations. Four treatments were performed as $1000 \mu \mathrm{M}, 625 \mu \mathrm{M}, 50 \mu \mathrm{M}$ and $10 \mu \mathrm{M}$ Pi. Statistics were performed by Duncan's test at the significance level of $P<0.05$. Values represent mean $\pm \mathrm{SD}, \mathrm{n}=4$ replicates of 20 seedlings each, Table S1: CRF family genes identified in Brassica napus, Table S2: Primer sequences used in this study

Author Contributions: Conceptualization, S.W. and G.D.; formal analysis, G.D. and H.Z.; investigation, G.D. and H.Z.; data curation, G.D.; writing—original draft preparation, S.W. and G.D.; writing-review and editing, L.S. and F.X.; supervision, G.D.; funding acquisition, G.D. All authors have read and agreed to the published version of the manuscript. 
Funding: This research was funded by the National Natural Science Foundation of China (31672215), the National Key Research and Development Program of China (2018YFD0200900), and the Fundamental Research Funds for the Central Universities of China (2662017QD039 and 2662019PY013).

Acknowledgments: We thank Chang Yang (from HZAU) and Hongyuan Du (from HZAU) for their contributions in performing the experiments.

Conflicts of Interest: The authors declare no conflict of interest. The funders had no role in the design of the study; in the collection, analyses, or interpretation of data; in the writing of the manuscript, or in the decision to publish the results.

\section{Abbreviations}

$\begin{array}{ll}\mathrm{P} & \text { Phosphorus } \\ \mathrm{Pi} & \text { Phosphate } \\ \mathrm{TF} & \text { Transcription factor } \\ \mathrm{OE} & \text { Overexpression } \\ \mathrm{PR} & \text { Primary root } \\ \text { LR } & \text { Lateral root } \\ \text { CRF } & \text { Cytokinin Response Factor } \\ \text { AP2 } & \text { APETALA2 } \\ \text { ERF } & \text { ETHYLENE RESPONSIVE FACTOR } \\ \text { FPKM } & \text { fragments per kilobase of transcript per million fragments mapped }\end{array}$

\section{References}

1. Vance, C.P.; Uhde-Stone, C.; Allan, D.L. Phosphorus acquisition and use: Critical adaptations by plants for securing a nonrenewable resource. New Phytol. 2003, 157, 423-447. [CrossRef]

2. Oldroyd, G.; Leyser, O. A plant's diet, surviving in a variable nutrient environment. Science 2020, 368, eaba0196. [CrossRef]

3. Liang, C.; Wang, J.; Zhao, J.; Tian, J.; Liao, H. Control of phosphate homeostasis through gene regulation in crops. Curr. Opin. Plant Biol. 2014, 21, 59-66. [CrossRef]

4. López-Arredondo, D.L.; Leyva-González, M.A.; González-Morales, S.I.; López-Bucio, J.; Herrera-Estrella, L. Phosphate nutrition: Improving low-phosphate tolerance in crops. Annu. Rev. Plant Biol. 2014, 65, 95-123. [CrossRef]

5. Shin, H.; Shin, H.S.; Dewbre, G.R.; Harrison, M.J. Phosphate transport in Arabidopsis: Pht1;1 and Pht1;4 play a major role in phosphate acquisition from both low- and high-phosphate environments. Plant J. 2004, 39, 629-642. [CrossRef]

6. Bari, R.; Datt Pant, B.; Stitt, M.; Scheible, W.R. PHO2, microRNA399, and PHR1 define a phosphate-signaling pathway in plants. Plant Physiol. 2006, 141, 988-999. [CrossRef]

7. Chiou, T.J.; Aung, K.; Lin, S.I.; Wu, C.C.; Chiang, S.F.; Su, C.L. Regulation of phosphate homeostasis by MicroRNA in Arabidopsis. Plant Cell 2006, 18, 412-421. [CrossRef] [PubMed]

8. Liu, T.Y.; Huang, T.K.; Tseng, C.Y.; Lai, Y.S.; Lin, S.I.; Lin, W.Y.; Chen, J.W.; Chiou, T.J. PHO2-dependent degradation of PHO1 modulates phosphate homeostasis in Arabidopsis. Plant Cell 2012, 24, 2168-2183. [CrossRef] [PubMed]

9. Essigmann, B.; Güler, S.; Narang, R.A.; Linke, D.; Benning, C. Phosphate availability affects the thylakoid lipid composition and the expression of $S Q D 1$, a gene required for sulfolipid biosynthesis in Arabidopsis thaliana. Proc. Natl. Acad. Sci. USA 1998, 95, 1950-1955. [CrossRef] [PubMed]

10. Härtel, H.; Dormann, P.; Benning, C. DGD1-independent biosynthesis of extraplastidic galactolipids after phosphate deprivation in Arabidopsis. Proc. Natl. Acad. Sci. USA 2000, 97, 10649-10654. [CrossRef] [PubMed]

11. Li, M.; Welti, R.; Wang, X. Quantitative profiling of Arabidopsis polar glycerolipids in response to phosphorus starvation. Roles of phospholipases $\mathrm{D} \zeta 1$ and $\mathrm{D} \zeta 2$ in phosphatidylcholine hydrolysis and digalactosyldiacylglycerol accumulation in phosphorus-starved plants. Plant Physiol. 2006, 142, 750-761. [CrossRef]

12. Svistoonoff, S.; Creff, A.; Reymond, M.; Sigoillot-Claude, C.; Ricaud, L.; Blanchet, A.; Nussaume, L.; Desnos, T. Root tip contact with low-phosphate media reprograms plant root architecture. Nat. Genet. 2007, 39, 792-796. [CrossRef] [PubMed] 
13. Camacho-Cristóbal, J.J.; Rexach, J.; Conéjéro, G.; Al-Ghazi, Y.; Nacry, P.; Doumas, P. PRD, an Arabidopsis AINTEGUMENTA-like gene, is involved in root architectural changes in response to phosphate starvation. Planta 2008, 228, 511-522. [CrossRef] [PubMed]

14. Rubio, V.; Linhares, F.; Solano, R.; Martín, A.C.; Iglesias, J.; Leyva, A.; Paz-Ares, J. A conserved MYB transcription factor involved in phosphate starvation signaling both in vascular plants and in unicellular algae. Genes Dev. 2001, 15, 2122-2133. [CrossRef] [PubMed]

15. Bustos, R.; Castrillo, G.; Linhares, F.; Puga, M.I.; Rubio, V.; Pérez-Pérez, J.; Solano, R.; Leyva, A.; Paz-Ares, J. A central regulatory system largely controls transcriptional activation and repression responses to phosphate starvation in Arabidopsis. PLoS Genet. 2010, 6, e1001102. [CrossRef] [PubMed]

16. Rashotte, A.M.; Mason, M.G.; Hutchison, C.E.; Ferreira, F.J.; Schaller, G.E.; Kieber, J.J. A subset of Arabidopsis AP2 transcription factors mediates cytokinin responses in concert with a two-component pathway. Proc. Natl. Acad. Sci. USA 2006, 103, 11081-11085. [CrossRef] [PubMed]

17. Kim, J. CYTOKININ RESPONSE FACTORs gating environmental signals and hormones. Trends Plant Sci. 2016, 21, 993-996. [CrossRef]

18. Rashotte, A.M.; Goertzen, L.R. The CRF domain defines cytokinin response factor proteins in plants. BMC Plant Biol. 2010, 10, 74. [CrossRef]

19. Ramaiah, M.; Jain, A.; Raghothama, K.G. ETHYLENE RESPONSE FACTOR070 regulates root development and phosphate starvation-mediated responses. Plant Physiol. 2014, 164, 1484-1498. [CrossRef]

20. Jeon, J.; Cho, C.; Lee, M.R.; Van Binh, N.; Kim, J. CYTOKININ RESPONSE FACTOR2 (CRF2) and CRF3 regulate lateral root development in response to cold stress in Arabidopsis. Plant Cell 2016, 28, 1828-1843. [CrossRef]

21. Chalhoub, B.; Denoeud, F.; Liu, S.; Parkin, I.A.; Tang, H.; Wang, X.; Chiquet, J.; Belcram, H.; Tong, C.; Samans, B.; et al. Early allopolyploid evolution in the post-Neolithic Brassica napus oilseed genome. Science 2014, 345, 950-953. [CrossRef]

22. Kong, L.; Zhao, K.; Gao, Y.; Miao, L.; Chen, C.; Deng, H.; Liu, Z.; Yu, X. Comparative analyses of cytokinin response factors in Brassica diploids and amphidiploids and insights into the evolution of Brassica species. BMC Genomics 2018, 19, 728. [CrossRef]

23. Bailey, T.L.; Boden, M.; Buske, F.A.; Frith, M.; Grant, C.E.; Clementi, L.; Ren, J.; Li, W.W.; Noble, W.S. MEME SUITE: Tools for motif discovery and searching. Nucleic Acids Res. 2009, 37, W202-W208. [CrossRef]

24. Brooks, M.D.; Cirrone, J.; Pasquino, A.V.; Alvarez, J.M.; Swift, J.; Mittal, S.; Juang, C.L.; Varala, K.; Gutiérrez, R.A.; Krouk, G.; et al. Network Walking charts transcriptional dynamics of nitrogen signaling by integrating validated and predicted genome-wide interactions. Nat. Commun. 2019, 10, 1569. [CrossRef]

25. Hammond, J.P.; Bennett, M.J.; Bowen, H.C.; Broadley, M.R.; Eastwood, D.C.; May, S.T.; Rahn, C.; Swarup, R.; Woolaway, K.E.; White, P.J. Changes in gene expression in Arabidopsis shoots during phosphate starvation and the potential for developing smart plants. Plant Physiol. 2003, 132, 578-596. [CrossRef]

26. Misson, J.; Raghothama, K.G.; Jain, A.; Jouhet, J.; Block, M.A.; Bligny, R.; Ortet, P.; Creff, A.; Somerville, S.; Rolland, N.; et al. A genome-wide transcriptional analyses using Arabidopsis thaliana Affymetrix gene chips determined plant responses to phosphate deprivation. Proc. Natl. Acad. Sci. USA 2005, 102, 11934-11939. [CrossRef]

27. Lin, W.D.; Liao, Y.Y.; Yang, T.J.; Pan, C.Y.; Buckhout, T.J.; Schmidt, W. Coexpression-based clustering of Arabidopsis root genes predicts functional modules in early phosphate deficiency signaling. Plant Physiol. 2011, 155, 1383-1402. [CrossRef]

28. O'Rourke, J.A.; Yang, S.S.; Miller, S.S.; Bucciarelli, B.; Liu, J.; Rydeen, A.; Bozsoki, Z.; Uhde-Stone, C.; Tu, Z.J.; Allan, D.; et al. An RNA-Seq transcriptome analyses of orthophosphate-deficient white lupin reveals novel insights into phosphorus acclimation in plants. Plant Physiol. 2013, 161, 705-724. [CrossRef]

29. Xue, Y.; Zhuang, Q.; Zhu, S.; Xiao, B.; Liang, C.; Liao, H.; Tian, J. Genome wide transcriptome analyses reveals complex regulatory mechanisms underlying phosphate homeostasis in soybean nodules. Int. J. Mol. Sci. 2018, 19, 2924. [CrossRef]

30. Shi, X.; Gupta, S.; Rashotte, A.M. Solanum lycopersicum cytokinin response factor (SlCRF) genes: Characterization of CRF domain-containing ERF genes in tomato. J. Exp. Bot. 2012, 63, 973-982. [CrossRef]

31. Gupta, S.; Rashotte, A.M. Expression patterns and regulation of SICRF3 and SICRF5 in response to cytokinin and abiotic stresses in tomato (Solanum lycopersicum). J. Plant Physiol. 2014, 171, 349-358. [CrossRef] 
32. Zwack, P.J.; De Clercq, I.; Howton, T.C.; Hallmark, H.T.; Hurny, A.; Keshishian, E.A.; Parish, A.M.; Benkova, E.; Mukhtar, M.S.; Van Breusegem, F.; et al. Cytokinin response factor 6 represses cytokinin-associated genes during oxidative stress. Plant Physiol. 2016, 172, 1249-1258. [CrossRef]

33. Cucinotta, M.; Manrique, S.; Guazzotti, A.; Quadrelli, N.E.; Mendes, M.A.; Benkova, E.; Colombo, L. Cytokinin response factors integrate auxin and cytokinin pathways for female reproductive organ development. Development 2016, 143, 4419-4424. [CrossRef]

34. Raines, T.; Shanks, C.; Cheng, C.Y.; McPherson, D.; Argueso, C.T.; Kim, H.J.; Franco-Zorrilla, J.M.; López-Vidriero, I.; Solano, R.; Vaňková, R.; et al. The cytokinin response factors modulate root and shoot growth and promote leaf senescence in Arabidopsis. Plant J. 2016, 85, 134-147. [CrossRef]

35. Qin, L.; Wang, L.; Guo, Y.; Li, Y.; Ümüt, H.; Wang, Y. An ERF transcription factor from Tamarix hispida, ThCRF1, can adjust osmotic potential and reactive oxygen species scavenging capability to improve salt tolerance. Plant Sci. 2017, 265, 154-166. [CrossRef]

36. Varala, K.; Marshall-Colón, A.; Cirrone, J.; Brooks, M.D.; Pasquino, A.V.; Léran, S.; Mittal, S.; Rock, T.M.; Edwards, M.B.; Kim, G.J.; et al. Temporal transcriptional logic of dynamic regulatory networks underlying nitrogen signaling and use in plants. Proc. Natl. Acad. Sci. USA 2018, 115, 6494-6499. [CrossRef]

37. Zheng, Z.; Wang, Z.; Wang, X.; Liu, D. Blue light-triggered chemical reactions underlie phosphate deficiency-induced inhibition of root elongation of Arabidopsis seedlings grown in Petri dishes. Mol. Plant. 2019, 12, 1515-1523. [CrossRef]

38. Letunic, I.; Doerks, T.; Bork, P. SMART: Recent updates, new developments and status in 2015. Nucleic Acids Res. 2015, 43, D257-D260. [CrossRef]

39. Marchler-Bauer, A.; Derbyshire, M.K.; Gonzales, N.R.; Lu, S.; Chitsaz, F.; Geer, L.Y.; Geer, R.C.; He, J.; Gwadz, M.; Hurwitz, D.I.; et al. CDD: NCBI's conserved domain database. Nucleic Acids Res. 2015, 43, D222-D226. [CrossRef]

40. Gasteiger, E.; Gattiker, A.; Hoogland, C.; Ivanyi, I.; Appel, R.D.; Bairoch, A. ExPASy: The proteomics server for in-depth protein knowledge and analyses. Nucleic Acids Res. 2003, 31, 3784-3788. [CrossRef]

41. Horton, P.; Park, K.J.; Obayashi, T.; Fujita, N.; Harada, H.; Adams-Collier, C.J.; Nakai, K. WoLF PSORT: Protein localization predictor. Nucleic Acids Res. 2007, 35, W585-W587. [CrossRef]

42. Chenna, R.; Sugawara, H.; Koike, T.; Lopez, R.; Gibson, T.J.; Higgins, D.G.; Thompson, J.D. Multiple sequence alignment with the Clustal series of programs. Nucleic Acids Res. 2003, 31, 3497-3500. [CrossRef]

43. Tamura, K.; Stecher, G.; Peterson, D.; Filipski, A.; Kumar, S. MEGA6: Molecular Evolutionary Genetics Analyses version 6.0. Mol. Biol. Evol. 2013, 30, 2725-2729. [CrossRef]

44. Krzywinski, M.; Schein, J.; Birol, I.; Connors, J.; Gascoyne, R.; Horsman, D.; Jones, S.J.; Marra, M.A. Circos: An information aesthetic for comparative genomics. Genome Res. 2009, 19, 1639-1645. [CrossRef]

45. Wang, Y.; Tang, H.; Debarry, J.D.; Tan, X.; Li, J.; Wang, X.; Lee, T.H.; Jin, H.; Marler, B.; Guo, H.; et al. MCScanX: A toolkit for detection and evolutionary analyses of gene synteny and collinearity. Nucleic Acids Res. 2012, 40, e49. [CrossRef]

46. Duan, H.Y.; Shi, L.; Ye, X.S.; Wang, Y.H.; Xu, F.S. Identification of phosphorous efficient germplasm in oilseed rape. J. Plant Nutr. 2009, 32, 1148-1163. [CrossRef]

47. Li, Y.; Wang, X.; Zhang, H.; Wang, S.; Ye, X.; Shi, L.; Xu, F.; Ding, G. Molecular identification of the phosphate transporter family 1 (PHT1) genes and their expression profiles in response to phosphorus deprivation and other abiotic stresses in Brassica napus. PLoS ONE 2019, 14, 0220374. [CrossRef]

48. Trapnell, C.; Roberts, A.; Goff, L.; Pertea, G.; Kim, D.; Kelley, D.R.; Pimentel, H.; Salzberg, S.L.; Rinn, J.L.; Pachter, L. Differential gene and transcript expression analysis of RNA-seq experiments with TopHat and Cufflinks. Nat. Protoc. 2012, 7, 562-578. [CrossRef]

49. Clough, S.J.; Bent, A.F. Floral dip: A simplified method for Agrobacterium-mediated transformation of Arabidopsis thaliana. Plant J. 1998, 16, 735-743. [CrossRef]

50. Pidkowich, M.S.; Nguyen, H.T.; Heilmann, I.; Ischebeck, T.; Shanklin, J. Modulating seed beta-ketoacyl-acyl carrier protein synthase II level converts the composition of a temperate seed oil to that of a palm-like tropical oil. Proc. Natl. Acad. Sci. USA 2007, 104, 4742-4747. [CrossRef] 